Portland State University

PDXScholar

Chemistry Faculty Publications and

Presentations

Chemistry

3-15-2000

\title{
Two-dimensional Fermi liquid with fixed chemical potential
}

J. Wosnitza

S. Wanka

J. Hagel

E. Balthes

N. Harrison

See next page for additional authors

Follow this and additional works at: https://pdxscholar.library.pdx.edu/chem_fac

Part of the Physics Commons

Let us know how access to this document benefits you.

Citation Details

Wosnitza, J., Wanka, S., Hagel, J., Balthes, E., Harrison, N., Schlueter, J. A., Kini, A. M., Geiser, U., Mohtasham, J., Winter, R. W., Gard, G. L. (2000). Two-dimensional Fermi liquid with fixed chemical potential. Physical Review B, 61, 7384.

This Article is brought to you for free and open access. It has been accepted for inclusion in Chemistry Faculty Publications and Presentations by an authorized administrator of PDXScholar. Please contact us if we can make this document more accessible: pdxscholar@pdx.edu. 


\section{Authors}

J. Wosnitza, S. Wanka, J. Hagel, E. Balthes, N. Harrison, J. A. Schlueter, A. M. Kini, U. Geiser, Javid Mohtasham, Rolf Walter Winter, and Gary L. Gard 


\title{
Two-dimensional Fermi liquid with fixed chemical potential
}

\author{
J. Wosnitza, S. Wanka, and J. Hagel, \\ Physikalisches Institut, Universität Karlsruhe, 76128 Karlsruhe, Germany \\ E. Balthes \\ Grenoble High Magnetic Field Laboratory, MPI and C.N.R.S., 38042 Grenoble, France \\ N. Harrison \\ National High Magnetic Field Laboratory, LANL Los Alamos, New Mexico 87545 \\ J. A. Schlueter, A. M. Kini, and U. Geiser \\ Chemistry and Materials Science Divisions, Argonne National Laboratory, Argonne, Ilinois 60439 \\ J. Mohtasham, R. W. Winter, and G. L. Gard \\ Department of Chemistry, Portland State University, Portland, Oregon 97207
}

(Received 28 October 1999)

\begin{abstract}
de Haas-van Alphen measurements made on the organic metal $\beta "$-(BEDT-TTF) ${ }_{2} \mathrm{SF}_{5} \mathrm{CH}_{2} \mathrm{CF}_{2} \mathrm{SO}_{3}$ reveal the existence of an ideal two-dimensional (2D) Fermi surface, but rather than having the conventional sawtooth wave form that is normally observed in all other 2D electron gases, instead, an "inverse sawtooth" wave form is observed, which is to be expected when the chemical potential is pinned at a constant value. While this proves the existence of the theoretically predicted quasi-one-dimensional band, it further implies that this band has an exceptionally large density of states.
\end{abstract}

The observation of magnetic quantum oscillations in three-dimensional (3D) metals is well proven, ${ }^{1}$ within the framework of the Lifshitz-Kosevich (LK) theory, ${ }^{2}$ to be a powerful tool for obtaining important band-structure parameters by means of quantitative analyses of experimental data. This is in contrast to two-dimensional (2D) conductors in which a qualitatively different behavior is expected. For an ideal 2D metal, i.e., a metal with only one 2D Fermi surface (FS), at sufficiently high-magnetic fields, $B$, a sawtooth-like de Haas-van Alphen (dHvA) signal (with sharp drops of the magnetization $M$ for rising fields) is predicted. While an analytical formula for the $2 \mathrm{D}$ electron gases applicable when $\hbar \omega_{c} \gg k_{B} T$ (where $\omega_{c}=e B / m_{c}$ is the cyclotron frequency, $m_{c}$ is the effective cyclotron mass, $k_{B}$ is the Boltzmann constant, and $T$ is the temperature) was derived in Ref. 3, important damping effects, such as those arising from quasiparticle scattering, were not included. Such effects can considerably broaden the Landau levels and thereby mask the 2D behavior. Subsequently, a numerical model was developed, ${ }^{4}$ which included these damping effects, and also the effect of a background reservoir of states, which reduces the extent of the oscillations of the chemical potential $\mu$. However, neither of these models can be utilized with the same simplicity that is possible with the LK formula in $3 \mathrm{D}$ metals $^{2}$ for extracting band-structure parameters.

It has been a long-standing experimental challenge to verify the theoretical predictions for the wave-shape, field, and temperature dependence of magnetic quantum oscillations in 2D metals, yet only recently have different groups succeeded in measuring the magnetization of multilayer ${ }^{5}$ and single-layer ${ }^{6} 2 \mathrm{D}$ electron gases within $\mathrm{GaAs} / \mathrm{Al}_{x} \mathrm{Ga}_{1-x} \mathrm{As}$ heterostructures directly. Sawtooth-like dHvA oscillations were observed, indeed, with sharp drops in $M$ occurring with rising field in line with theoretical predictions, ${ }^{3,4}$ thereby confirming the validity of the canonical ensemble for a $2 \mathrm{D}$ electron gas. A much larger and, therefore, easier to measure dHvA signal has been observed in 2D layered organic metals. These materials are characterized by a highly 2D electronic band structure with, often, negligible dispersion (or warping of the cylindrical Fermi surface) in the third direction. ${ }^{7}$ In spite of the fact that these materials are of high quality with long scattering times $\tau$, i.e., small Dingle temperatures $T_{D}=\hbar / 2 \pi k_{B} \tau$, and the condition $\hbar \omega_{c} \gg k_{B} T$ is very easily reached, ${ }^{7}$ the dHvA oscillations in most of these bulk quasi-2D organic metals exhibit only marginal deviations from the 3D LK theory. The only notable exceptions are $\kappa$-(BEDT-TTF $)_{2} \mathrm{I}_{3}, \alpha-(\mathrm{BEDT}-\mathrm{TTF})_{2} \mathrm{KHg}(\mathrm{SCN})_{4}$, and $\Theta-(\text { BEDT-TTF })_{2} \mathrm{I}_{3}, 4,8,9$ where BEDT-TTF is bis-ethylenedithio-tetra-thia-fulvalene. In the latter salt, a sawtooth magnetization with very sharp drops was reported, ${ }^{8}$ yet, no quantitative analysis of the data was presented and the result was never reproduced. In $\kappa$-(BEDT-TTF $)_{2} \mathrm{I}_{3}$ at high-magnetic fields and low temperatures, a sawtooth magnetization with an apparently enhanced spin splitting and an apparently field-dependent effective mass could quantitatively be explained by the effect of the oscillations of the chemical potential in a 2D Fermi liquid. ${ }^{9}$ In other layered organic metals, such deviations are more subtle, with only a moderate enhancement of harmonic content and small reduction in their apparent effective masses having been observed. ${ }^{4,9-11}$

In contrast, very strong deviations from conventional LK behavior were reported to occur with magnetoresistance oscillations, i.e., for the Shubnikov-de Haas $(\mathrm{SdH})$ effect, in a 


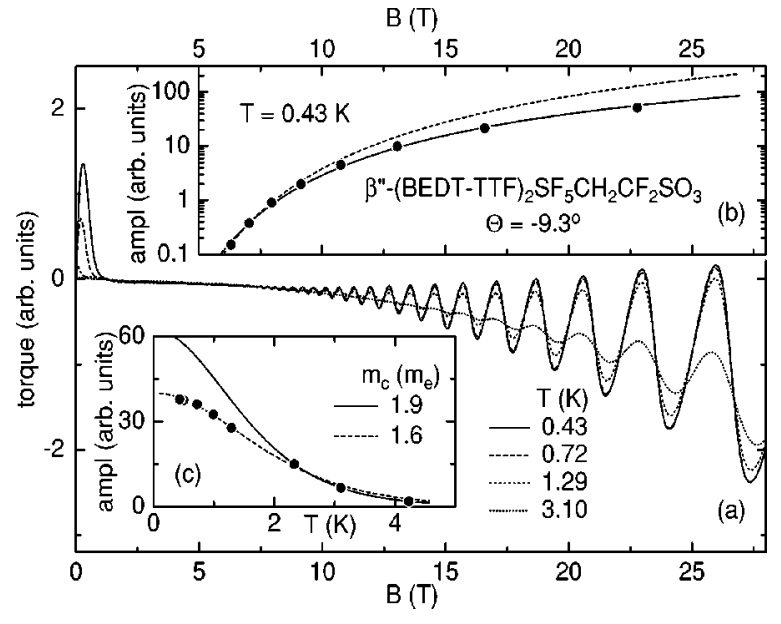

FIG. 1. (a) Field dependence of the torque signal of sample 1 for selected temperatures. (b) Field dependence of the dHvA amplitude at $T=0.43 \mathrm{~K}$. The lines show the expected field dependence from 3D LK theory (dashed line) and for the 2D case [solid line, see Eq. (1)]. (c) Temperature dependence of the dHvA amplitude for an average field of $19.8 \mathrm{~T}$. The dashed line is a fit with the LK theory (equivalent to that expected for the temperature dependence in the 2D grand canonical ensemble). The solid line shows the expected dependence for the effective mass determined at low fields.

number of organic metals. ${ }^{9,12-15}$ The applicability of the LK formula in the case of the $\mathrm{SdH}$ effect is, nevertheless, problematic owing to complications involving the conductivity tensor, and other novel effects that appear to be unique to magnetotransport. ${ }^{12,16}$ Hence, a reliable comparison of experimental results with theoretical predictions should be done with true thermodynamic quantities such as the magnetization. Here, we provide experimental evidence for a unique situation where the magnetic quantum oscillations of a 2D metal are qualitatively different from the theoretical expectation $^{3}$ and from the observed behavior in semiconductor-based 2D electron gases ${ }^{5,6}$ and bulk organic metals. ${ }^{4,8,9}$ Rather unusually, the measured dHvA signal has exactly the form to be expected for a system in which the chemical potential is pinned at a constant value, i.e., the $2 \mathrm{D}$ grand canonical ensemble. ${ }^{1,4}$

The single crystals of the organic superconductor $\beta^{\prime \prime}$-(BEDT-TTF) ${ }_{2} \mathrm{SF}_{5} \mathrm{CH}_{2} \mathrm{CF}_{2} \mathrm{SO}_{3}\left(T_{c} \approx 4.4 \mathrm{~K}\right)$ used here were grown by electrocrystallization. ${ }^{17}$ In contrast to earlier crystals $^{11,18}$ the quality of the samples here (which we shall refer to as 1 and 2) were much better, as evidenced by the lower Dingle temperatures (see below). The dHvA effect was measured by means of a capacitance cantilever torque magnetometer mounted on a rotatable sample holder in a ${ }^{3} \mathrm{He}$ cryostat at the Grenoble High Magnetic Field Laboratory in fields of up to $28 \mathrm{~T}$.

Figure 1 (a) shows the raw torque signal, $\vec{\tau}=\vec{M} \times \vec{B}$, of sample 1 at different temperatures. The pronounced peak below about $1.5 \mathrm{~T}$ originates from the diamagnetic response of the sample in its superconducting state. For rising fields, a type-II superconductor exhibits diamagnetism, i.e., a negative magnetization. For falling fields, a positive magnetization occurs below the irreversibility point, with the hysteresis being due to vortex pinning effects [see Fig. 2(c)]. Here, we discuss only the torque dHvA data taken on the rising field.

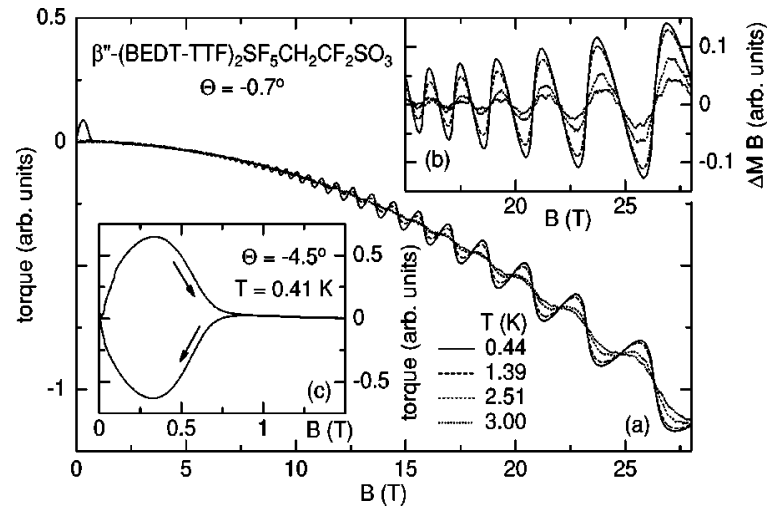

FIG. 2. (a) Field dependence of the torque signal of sample 2 at selected temperatures for $\Theta=-0.7^{\circ}$. (b) dHvA signal times $B$ extracted from the negative torque signal after subtraction of a quadratic background. (c) Low-field hysteresis of the torque signal at $\Theta=-4.5^{\circ}$ for rising and falling field.

The sign of the torque signal depends on the relative orientation of $B$ with respect to the cantilever, and on the sign of the capacitance change as the cantilever is bent. The observation of the diamagnetic peak in the low-field torque enables the unequivocal determination of the sign of $M$, which is important for the wave-shape analysis of the dHvA oscillations discussed in detail below.

The dHvA signal is clearly apparent as the oscillating part superimposed on a steady background of the torque, increasing with field. The steady part of the torque is proportional to $B^{2}$, arising from an anisotropic field-independent susceptibility $\chi$ of the sample. In general, $\chi$ is a tensor and the magnetization is proportional to the product of this tensor with $\vec{B}$. Thus, the absolute value of the torque has the form $\tau$ $\propto \Delta \chi B^{2} \sin \vartheta \cos \vartheta$, where $\Delta \chi$ is the susceptibility difference between two principal axes of the $\chi$ tensor and $\vartheta$ is the angle between $B$ and one of principal tensor axes. In the present material, the principal axes do not coincide with the crystal axes. One of these axes occurs at $\Theta \approx 30^{\circ}$, where $\Theta$ is the angle between $B$ and the normal to the conducting layers, as evidenced by the vanishing of the magnetic torque at this angle. The origin of this unusual anisotropic susceptibility is unclear, but might be a remnant of the local magnetism of the molecular bands.

The torque acting on a sample with an anisotropic FS is proportional to the derivative of the extremal area of the FS with respect to $\Theta$, i.e., proportional to $d F / d \Theta$, where $F$ $=F_{0} / \cos \Theta$ is the dHvA frequency of a $2 \mathrm{D}$ cylindrical FS. Hence, for $\Theta \approx 0$, the amplitude of the dHvA signal in the magnetic torque is rather small. This has been utilized in the measurement of sample 2 shown in Fig. 2(a) to exclude any nonlinearities of the torque signal due, e.g., to the torque interaction effect, and to ensure the reliability of the data. ${ }^{19}$ The steady part of the torque at $\Theta=-0.7^{\circ}$ follows perfectly the $B^{2}$ behavior up to the highest fields, implying that the torquemeter operates unequivocally in the linear regime and that the relatively small dHvA signal is undisturbed by experimental artifacts. Another possible distortion of the dHvA signal might in principal be caused by magnetic interaction. ${ }^{1}$ Here, this can only be of marginal importance owing to the small magnetization and the very small dHvA frequency.

The angular dependence of the dHvA frequency with $F_{0}$ 
$=198 \pm 1 \mathrm{~T}$ agrees closely with previous results. ${ }^{11,14,15,18}$ However, at low temperatures and high fields, clear deviations from the conventional 3D LK theory are observed in both samples. Figure 1(b) shows the field dependence of the fundamental dHvA amplitude extracted from the Fourier transformations made over short intervals in $1 / B$ of the data of sample 1 at $T=0.43 \mathrm{~K}$. The dashed line depicts the behavior expected for a 3D metal with $T_{D}=1.3 \mathrm{~K}$, as obtained from a best fit to the 3D LK formula at low-magnetic fields. At higher fields, the data lie clearly below the fitted line; note the logarithmic scale of the ordinate. A fit of the 3D LK formula at higher fields would yield a much lower $T_{D}$, but leaving the low-field data well below the fit line. A completely analogous behavior is observed for sample 2. A further observation is the apparent field-dependent effective cyclotron mass, $m_{c}$. At low fields, below $\sim 12 \mathrm{~T}, m_{c}$ $=1.9 m_{e}$ is found to be in good agreement with earlier lowfield data. ${ }^{18}$ At higher fields, however, the fit to the LK formula results in an appreciably reduced effective mass, i.e., $m_{c}=1.6 m_{e}$ for an average field of $B \approx 20 \mathrm{~T}$ [Fig. 1(c)]. A similar apparent mass reduction with increasing magnetic field was observed previously with $\mathrm{dHvA}$ data of another organic superconductor, where it could be explained by the influence of chemical-potential oscillations on the wave form in a $2 \mathrm{D}$ spin-split Fermi liquid. ${ }^{9}$ In the present case, however, we are far from the spin-split condition, and the origin of the apparent mass reduction is not clear (see discussion below). Another feature, which has been observed already for a number of 2D organic metals (see, e.g., Refs. 9 and 11), is that the higher harmonics of the dHvA signal are more weakly temperature-dependent than predicted by either the 3D LK theory or the 2D grand cananonical ensemble. This apparent behavior leads to very low estimates of $m_{c}$ for the higher harmonics. Here, we obtain $m_{c} \approx 1.2 m_{e}$ from a fit to the second harmonic of sample 1 at $B \approx 20 \mathrm{~T}$.

A remarkably new aspect of the data reported here, is the clear observation of an "inversed sawtooth" of the dHvA signal (having subtracted of the quadratic background [Fig. 2(b)]). Such a behavior is not expected for a $2 \mathrm{D}$ metal in the canonical ensemble, i.e., with fixed number of charge carriers and oscillating chemical potential. It is, however, exactly the wave shape predicted by the 2D grand canonical ensemble approximation, originally calculated for a FS slab in $k$ space assuming a fixed chemical potential. In this case, the magnetization is given simply by ${ }^{1}$

$$
M \propto-\sum_{r=1}^{\infty} \frac{1}{r} R_{D} R_{T} R_{S} \sin \left[2 \pi r\left(\frac{F}{B}-\frac{1}{2}\right)\right],
$$

with the Dingle $\left(R_{D}\right)$, temperature $\left(R_{T}\right)$, and spin-splitting damping factors $\left(R_{S}\right)$ being identical to those in the 3D LK formula. ${ }^{1}$ The only principal differences in the $2 \mathrm{D}$ formula are the omission of the $B^{1 / 2}$ factor, the omission of the $\pi / 4$ phase factor, and the modified harmonic content, namely a factor $r^{-1}$ in Eq. (1) occurs instead of $r^{-3 / 2}$ in the 3D formula. The first is responsible for the slower increase of the dHvA amplitude with field [see the solid line in Fig. 1(b)] compared to that expected from 3D LK theory. The latter two account for the Fourier components of the "inverse saw-

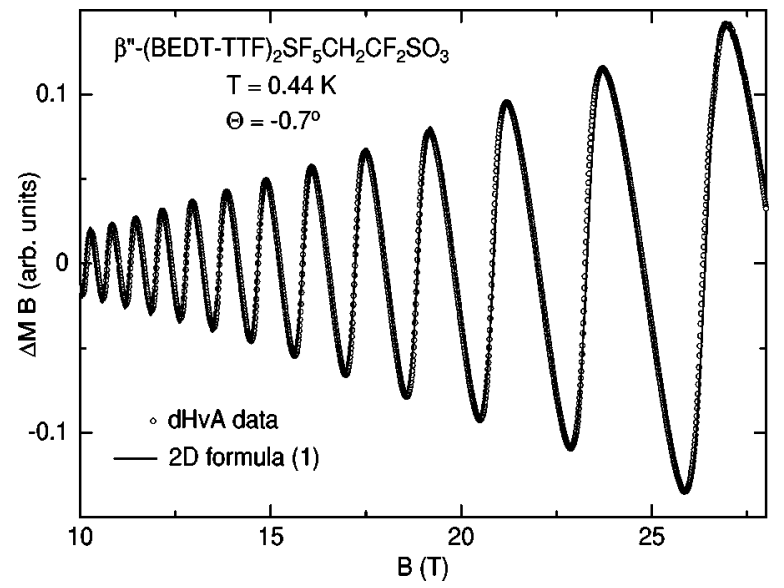

FIG. 3. Comparison of the measured dHvA data of sample 2 (open circles) with the 2D formula [Eq. (1), solid line] lying virtually on top of the experimental data.

tooth" wave form, characterized by an increased harmonic content and more steeply increasing slopes of the dHvA signal with respect to $B$.

The extraordinary ability of Eq. (1) to reproduce the experimental data is most clearly apparent for sample 2 (in Fig. 3 ), where the dHvA data are plotted as open circles enabling them to be distinguished from theory (solid line). The parameters used are those obtained from previous measurements ${ }^{11,18}$ and from the low-field high-temperature data of the present experiment, i.e., $m_{c}=1.9 m_{e}, g m_{c} / m_{e}$ $=3.9$ (which is independent from the theory employed), and $T_{D}=0.4 \mathrm{~K}$. Indeed, the latter parameter can be analytically extracted from the present data by means of fits to Eq. (1) and fits with the low-field estimate using the 3D LK formula. It is the exceptionally high quality of sample 2 that gives rise to the inversed-sawtooth shape of the dHvA signal, which then becomes less pronounced-but still detectable-for samples with higher scattering rates, i.e., for sample 1 with $T_{D}=1.3 \mathrm{~K}$. Note that, with the exception of a simple scaling factor, no free parameter is necessary to obtain the almost perfect agreement of data and 2D theory (1).

An important ingredient for the validity of Eq. (1) is the assumption of a fixed chemical potential, or equivalently, the existence of a charge-carrier reservoir. Band-structure calculations do after all predict the existence of a pair of quasi-1D electron surfaces ${ }^{18}$ in addition to the $2 \mathrm{D}$ hole pocket that gives rise to the $\mathrm{dHvA}$ signal. It is therefore most likely that it is this $1 \mathrm{D}$ band that acts as charge-carrier reservoir causing the chemical potential to be pinned. The influence of an additional density of states (DOS), originating from 1D bands, has been shown to modify the dHvA wave shape. ${ }^{4}$ Simulations of the dHvA signal with this numerical model show that the 1D DOS has to be at least approximately five times larger than the 2D DOS at the Fermi level. ${ }^{20}$ However, the numerical model reproduces the experimental data somewhat less accurately than Eq. (1). In particular, it is the steep slopes of the rising magnetization that cannot be reproduced perfectly by the numerical model.

Until now, there has been no direct evidence for the existence of these 1D bands in the present material. Only some rather indirect indications, such as a negligible Hall effect and the enormous background magnetoresistance, ${ }^{14,15}$ have 
been reported. The present results show clearly that $\beta^{\prime \prime}$-(BEDT-TTF) ${ }_{2} \mathrm{SF}_{5} \mathrm{CH}_{2} \mathrm{CF}_{2} \mathrm{SO}_{3}$ is a compensated metal with a large 1D DOS. This provides additional support for the validity of the predicted band structure and makes speculations on a possible density-wave transition questionable.

Although the expression for the 2D grand canonical ensemble reproduces the experimental dHvA data at low temperatures rather well, the temperature dependence of the signals, i.e., the apparent field dependence of $m_{c}$, cannot be explained. The 2D grand canonical ensemble predicts the same temperature dependence of the $\mathrm{dHvA}$ signal as the 3D formula. In the numerical model, a notable apparent fielddependent effective cyclotron mass is only expected close to a spin-splitting zero, ${ }^{9}$ which is not the case here. The origin for the reduced $m_{c}$ found for both samples, independent of quality, is unclear but might be caused by a crossover from the $2 \mathrm{D}$ theory at low temperatures to the $3 \mathrm{D}$ behavior at higher temperatures. It would be of considerable interest to investigate the origin of this aparent field-dependent $m_{c}$.

There is a debate on the actual Landau-level broadening due to impurity scattering; e.g., whether the Landau levels have Gaussian or Lorentzian line shapes (see Ref. 5 for references). The very clean dHvA signal in our case can be well reproduced by Eq. (1) assuming a Lorentzian boadening. As we checked by means of the numerical model, ${ }^{4}$ a fieldindependent Gaussian Landau-level profile gives a less good description of the experimental data since it results in a much weaker slope for the increasing branch of the dHvA signal.

The pronounced two dimensionality of the present material is equally reflected in strong anharmonicities of the mag- netoresistance oscillations at higher fields and lower temperatures. ${ }^{14,15,21}$ This is partially understood since the $\mathrm{SdH}$ effect, i.e., the relative conductivity oscillations, is expected to be proportional to $B^{2} d M / d B$. This leads to strongly peaked nonsinusoidal $\mathrm{SdH}$ signals, as have been observed experimentally. ${ }^{22}$ However, the large background magnetoresistance in strong fields, with an apparent fieldinduced insulating behavior, ${ }^{14,15}$ prohibits a reliable comparison of the experimental magnetoresistance oscillations with theory as is possible here for the dHvA effect.

In conclusion, we have found clear experimental evidence for a 2D Fermi liquid in a bulk organic material. In contrast to previously reported scenarios, we observed magnetic quantum oscillations that are characteristic of a 2D grand canonical ensemble, which can only be possible if the chemical potential is fixed. This, therefore, can be considered as proof for a field-independent fixed chemical potential, indicating the existence of a large 1D DOS at the Fermi energy of the present material.

We thank H. v. Löhneysen and M.-H. Whangbo for helpful discussions. This work was supported by the DFG and by the TMR Program under Contract No. ERBFMGECT950077. The National High Magnetic Field Laboratory is supported by NSF, the state of Florida, and the U.S. Dept. of Energy. Work at Argonne National Laboratory was supported by the U.S. Dept. of Energy (W-31-109-ENG38). Work at Portland State University was supported by NSF (Che-9632815) and the Petroleum Research Fund (ACS-PRF \# 31099-AC1).
${ }^{1}$ D. Shoenberg, Magnetic Oscillations in Metals (Cambridge University Press, Cambridge, 1984).

${ }^{2}$ M. Lifshitz and A.M. Kosevich, Zh. Éksp. Teor. Fiz. 29, 730 (1956) [Sov. Phys. JETP 2, 636 (1956)].

${ }^{3}$ I.D. Vagner, T. Maniv, and E. Ehrenfreund, Phys. Rev. Lett. 51, 1700 (1983); K. Jauregi, V.I. Marchenko, and I.D. Vagner, Phys. Rev. B 41, 12922 (1990).

${ }^{4}$ N. Harrison, R. Bogaerts, P.H.P. Reinders, J. Singleton, S.J. Blundell, and F. Herlach, Phys. Rev. B 54, 9977 (1996); J. Singleton, N. Harrison, R. Bogaerts, P.H.P. Reinders, I. Deckers, S.J. Blundell, and F. Herlach, Synth. Met. 86, 1907 (1997).

${ }^{5}$ A. Potts, R. Shepherd, W.G. Herrenden-Harker, M. Elliot, C.L. Jones, A. Usher, G.A.C. Jones, D.A. Ritchie, E.H. Linfield, and M. Grisham, J. Phys.: Condens. Matter 8, 5189 (1996).

${ }^{6}$ S.A.J. Wiegers, M. Specht, L.P. Lévy, M.Y. Simmons, D.A. Ritchie, A. Cavanna, B. Etienne, G. Martinez, and P. Wyder, Phys. Rev. Lett. 79, 3238 (1997).

${ }^{7}$ J. Wosnitza, Fermi Surfaces of Low-Dimensional Organic Metals and Superconductors (Springer, Berlin, 1996).

${ }^{8}$ M. Tokumoto, A.G. Swanson, J.S. Brooks, M. Tamura, H. Tajima, and H. Kuroda, Solid State Commun. 75, 439 (1990).

${ }^{9}$ N. Harrison, C.H. Mielke, D.G. Rickel, J. Wosnitza, J.S. Qualls, J.S. Brooks, E. Balthes, D. Schweitzer, I. Heinen, and W. Strunz, Phys. Rev. B 58, 10248 (1998).

${ }^{10}$ D. Beckmann, S. Wanka, J. Wosnitza, G. Goll, B. Drehmann, E. Balthes, D. Schweitzer, and W. Strunz, Eur. Phys. J. B 6, 329 (1998).
${ }^{11}$ J. Wosnitza, G. Goll, D. Beckmann, S. Wanka, J.A. Schlueter, J.M. Williams, P.G. Nixon, R.W. Winter, and G.L. Gard, Physica B 246-247, 104 (1998).

${ }^{12}$ E. Balthes, D. Schweitzer, I. Heinen, H.J. Keller, W. Strunz, W. Biberacher, A.G.M. Jansen, and E. Steep, Z. Phys. B: Condens. Matter 99, 163 (1996); E. Balthes, M. Schiller, D. Schweitzer, I. Heinen, W. Strunz, E. Steep, A.G.M. Jansen, and P. Wyder, Europhys. Lett. 47, 70 (1999).

${ }^{13}$ V.N. Laukhin, A. Audouard, H. Rakoto, J.M. Broto, F. Goze, G. Coffe, L. Brossard, J.P. Redoules, M.V. Kartsovnik, N.D. Kushch, L.I. Buravov, A.G. Khomenko, É.B. Yagubskii, S. Askenazy, and P. Pari, Physica B 211, 282 (1995); P.S. Sandhu, G.J. Athas, J.S. Brooks, E.G. Haanappel, J.D. Goettee, D.W. Rickel, M. Tokumoto, N. Kinoshita, and Y. Tanaka, Surf. Sci. 361-362, 913 (1996).

${ }^{14}$ J. Wosnitza, S. Wanka, J.S. Qualls, J.S. Brooks, C.H. Mielke, N. Harrison, J.A. Schlueter, J.M. Williams, P.G. Nixon, R.W. Winter, and G.L. Gard, Synth. Met. 103, 2000 (1999).

${ }^{15}$ F. Zuo, X. Su, P. Zhang, J.S. Brooks, J. Wosnitza, J.A. Schlueter, J.M. Williams, P.G. Nixon, R.W. Winter, and G.L. Gard, Phys. Rev. B 60, 6296 (1999).

${ }^{16}$ S. Hill, P.S. Sandhu, J.S. Qualls, J.S. Brooks, M. Tokumoto, N. Kinoshita, T. Kinoshita, and Y. Tanaka, Phys. Rev. B 55, R4891 (1997); M.M. Honold, N. Harrison, J. Singleton, H. Yaguchi, C. Mielke, D. Rickel, L. Deckers, P.H.P. Reinders, F. Herlach, M. Kurmoo, and P. Day, J. Phys.: Condens. Matter 9, L533 (1997).

${ }^{17}$ U. Geiser, J.A. Schlueter, H.H. Wang, A.M. Kini, J.M. Williams, 
P.P. Sche, H.I. Zakowicz, M.L. VanZile, J.D. Dudek, P.G. Nixon, R.W. Winter, G.L. Gard, J. Ren, and M.-H. Whangbo, J. Am. Chem. Soc. 118, 9996 (1996); J.A. Schlueter, U. Geiser, J.M. Williams, J.D. Dudek, M.E. Kelly, J.P. Flynn, R.R. Wilson, H.I. Zakowicz, P.P. Sche, D. Naumann, T. Roy, P.G. Nixon, R.W. Winter, and G.L. Gard, Synth. Met. 85, 1453 (1997).

${ }^{18}$ D. Beckmann, S. Wanka, J. Wosnitza, J.A. Schlueter, J.M. Williams, P.G. Nixon, R.W. Winter, G.L. Gard, J. Ren, and M.-H. Whangbo, Eur. Phys. J. B 1, 295 (1998).

${ }^{19}$ Torque interaction was indeed observed for another sample in a separate experiment. This resulted in a steepening of the falling side of the dHvA signal.

${ }^{20}$ Higher values than five for the 1D DOS result in only marginal changes of the dHvA signal.

${ }^{21}$ The low-field SdH data in Ref. 18 are still in line with the conventional 3D LK theory.

${ }^{22}$ J. Wosnitza, S. Wanka, J. Hagel, J. S. Qualls, J. S. Brooks, J. A. Schlueter, A. M. Kini, U. Geiser, J. Mohtasham, R. W. Winter, and G. L. Gard. (unpublished). 